\title{
Insuficiencia cardíaca como presentación de sarcoma cardíaco. Caso clínico y revisión de la literatura
}

\author{
Iván Cañete ${ }^{1, a}$, Felipe Carrillo ${ }^{1, a}$, Gabriel Uribe ${ }^{1, a}$, Juan Garcia ${ }^{2, c}$, , Marcelo Llancaqueo ${ }^{2, b}$. \\ 1. Departamento De Medicina Interna, Hospital Clínico Universidad De Chile, Universidad De Chile. Santiago. Chile. \\ 2. Departamento De Cardiología Y Cirugía Cardiovascular, Hospital Clínico Universidad De Chile. Santiago. Chile. \\ a. Médico Residente Medicina Interna, Universidad De Chile, Hospital Clínico Universidad De Chile, Santiago. Chile. \\ b. Cardiólogo, Hospital Clínico Universidad De Chile. Santiago. Chile. \\ c. Médico Residente Cardiología, Universidad De Chile, Hospital Clínico Universidad De Chile, Santiago. Chile. \\ Los autores no declaran tener conflictos de interés. \\ No se recibió financiamiento.
}

Los tumores cardíacos primarios malignos son infrecuentes en la práctica clínica. Suelen ser oligosintomáticos y de diagnóstico tardío lo que tiene como consecuencia una alta mortalidad. El caso clínico que se presenta a continuación es un ejemplo de esta patología, que debe ser especialmente considerada cuando la insuficiencia cardíaca no tiene una etiología clara.
Se describen los hallazgos clínicos y de laboratorio, como también los resultados de estudios de imágenes y la histología. Se ilustra los hallazgos en la operación y la evolución clínica del paciente. Se incluye una revisión de la literatura.

Palabras clave: tumor, sarcoma, insuficiencia cardíaca.

\section{Cardiac sarcoma associated to heart failure: Case report and review}

Malignant primary cardiac tumors are infrequent in clinical practice. They are usually oligosymptomatic and a late diagnosis leads to a high mortality rate. The clinical case of a patient presenting with heart failure with unclear etiology is presented. Clinical and laboratory findings are described along with the results of imaging studies. Findings at the time of surgery and histopathological characteristics are illustrated. A review of the literature is included. Key words: Tumor; cardiac sarcoma; heart failure.

Correspondencia: 


\section{Caso clínico:}

Hombre de 78 años, con antecedente de hipertensión arterial diagnosticada hace 8 años y buena adherencia a tratamiento. Su capacidad funcional era I.

Cursa con 1 mes de disnea de instalación insidiosa y progresiva asociada a edema de extremidades inferiores de predominio vespertino. En las últimas 48 horas evolucionó con ortopnea y acentuación de la disnea. Niega angina, síncope, palpitaciones, nicturia, disnea paroxística nocturna, síntomas respiratorios, fiebre o baja de peso.

Presentaba taquicardia (120 latidos por minuto), con demás signos vitales normales. Al examen físico presentaba ingurgitación yugular a $45^{\circ}$, crepitaciones pulmonares bibasales con murmullo atenuado, ritmo cardíaco regular, sin soplos y edema de extremidades inferiores. En el estudio inicial destacaba anemia con hemoglobina $12.8 \mathrm{gr} / \mathrm{dl}$, normocítica, normocrómica, plaquetas de $20.000 \mathrm{x} \mathrm{mm3} \mathrm{(VN:} \mathrm{150.000-400.000),}$ LDH 880 (VN: 120-246), TTPa 40 segundos (VN 2535 segundos), INR 1.5, Dímero D 7676 (VN: <500), Fibrinógeno 180 (VN: 180-350). El electrocardiograma mostró ritmo sinusal con complejos QRS disminuidos de voltaje, sin alteraciones de repolarización.

Se decide hospitalizar, complementando el estudio con TC de tórax que muestra derrame pleural bilateral asociado a patrón vidrio esmerilado. El ecocardiograma transtorácico informó hipertensión pulmonar moderada (53 $\mathrm{mmHg}$ ), aurícula izquierda levemente dilatada, ventrículo izquierdo de dimensiones y función sistólica conservada. (Figura 1).

Evoluciona con disnea de reposo por lo que se descarta tromboembolismo mediante angiotomografía. En el ecocardiograma torácico y trans esofágico se informan 3 masas en la aurícula izquierda, que se extienden a la desembocadura de las venas pulmonares y protruyen hacia ventrículo izquierdo, siendo la mayor de 6 por 4 centímetros y ocupando aproximadamente $80 \%$ de la cámara. No se realizó RNM de corazón.

Se interpreta el cuadro como IC secundario a tumor cardíaco, y se procede a la extirpación tumoral con plastía de septum y venas pulmonares derechas. Durante la cirugía se observa un gran tumor con consistencia y apariencia diferente de un mixoma, que ocupa la totalidad de la cavidad auricular izquierda. Se extiende a las 4 venas pulmonares, a lo menos un par de centímetros, comprometiendo parcialmente su pared. Existe una extensión del tumor al aparato subvalvular mitral, que se ve engrosado.

La biopsia fue informada como "Neoplasia mesenquimática maligna, principalmente, fusocelular y áreas de disposición epitelioide con células de pleomorfismo acentuado, núcleos hipercromáticos, algunos bizarros y recuento mitóticos de hasta 20 mitosis por 10 campos de mayor aumento". Existía una necrosis tumoral mayor al $50 \%$. El diagnóstico patológico final fue sarcoma de alto grado indiferenciado (Figura 2).

Evolucionó con paresia braquial derecha y cuadrantopsia homónima derecha y disminución del hemicampo visual derecho. La TC cerebro mostró hematoma paren-

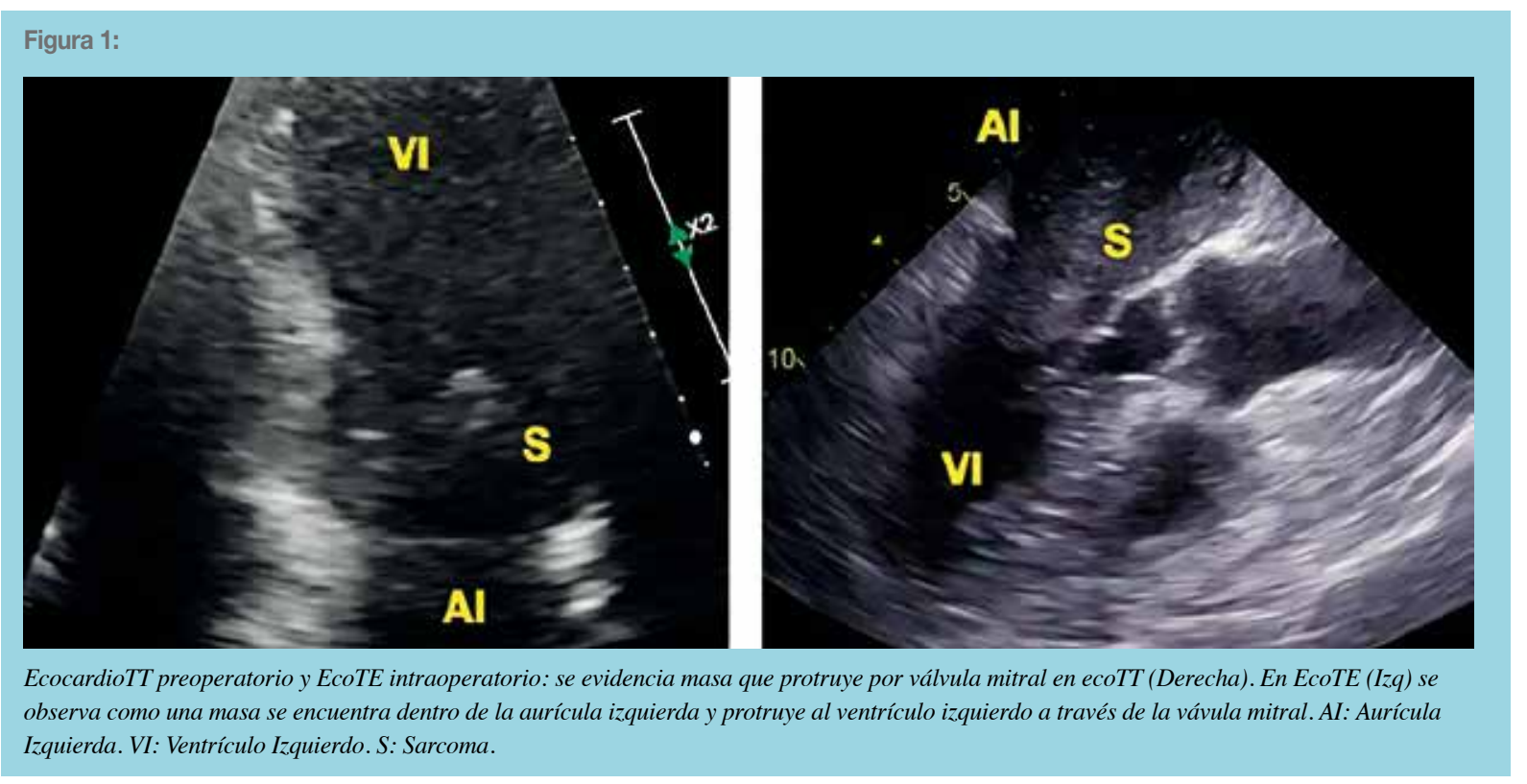




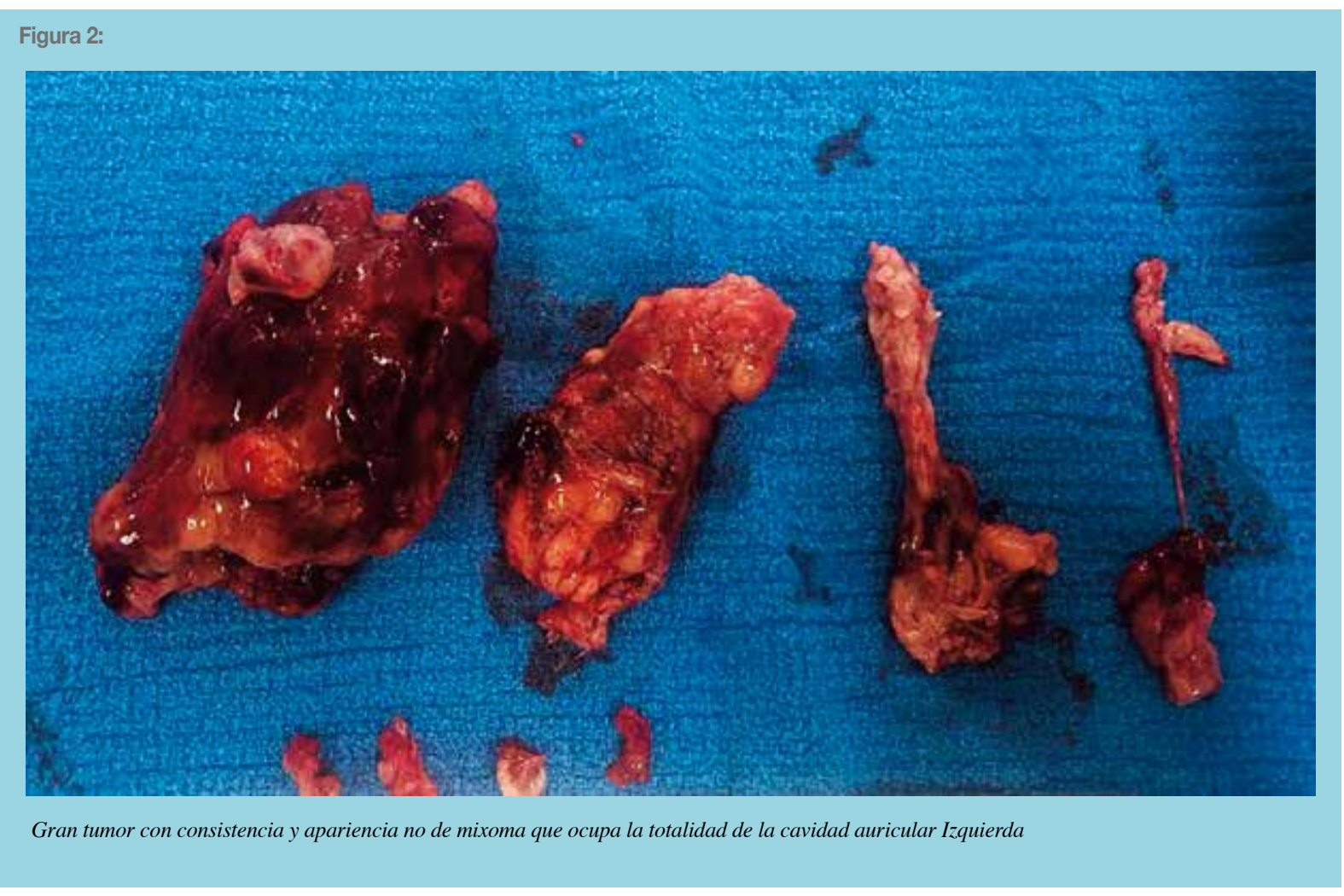

quimatoso occipital izquierdo con edema vasogénico. Se complementa el estudio con RNM de cerebro que revela lesión hemorrágica occipital izquierda con compromiso córtico-meníngeo focal y diseminado, compatibles con implantes secundarios occipitales a nivel supra e infra tentorial. No existía posibilidad de resección tumoral.

\section{Discusión:}

Los tumores cardíacos primarios son una patología de baja prevalencia y de difícil diagnóstico, el cual se realiza habitualmente en retrospectiva, post mortem ${ }^{1,2}$.

El 75\% de los tumores son benignos, siendo el mixoma el más frecuente con hasta el $50 \%$ de los casos reporta$\operatorname{dos}^{3}$. De los malignos, los sarcomas dan cuenta del $75 \%$ al $95 \%$ de los $\operatorname{casos}^{9,10}$. En Chile, la revisión de González et al, describe 72 tumores cardíacos, 49 (68\% de los casos) correspondían a mixomas y $6(8,3 \%)$ a sarcomas, 3 de ellos de alto grado ${ }^{2}$.

En cuanto a la incidencia, no existe diferencias significativas entre $\operatorname{sexos}^{4,5}$. La edad de presentación más común es entre los 40 y 50 años ${ }^{4,5,6}$, y es un factor pronóstico relevante.

El principal síntoma descrito es la disnea, cuya progre- sión dependerá de la velocidad de crecimiento del tumor $^{4,5,6}$. Otros síntomas descritos son: dolor torácico, arritmias, edema periférico, disnea, ortopnea y taponamiento $^{5}$. En el caso presentado, el motivo de consulta fue la disnea de instauración aguda, que podría explicarse por el tamaño tumoral y la ocupación en la cavidad cardíaca, siendo provocado por agregación plaquetaria, generando además trombocitopenia. Los síntomas extratorácicos asociados al sarcoma cardíaco aparecen según la velocidad de instauración de los síntomas, así como también la ubicación del tumor y la presencia o no de metástasis. Las metástasis cerebrales de sarcomas cardíacos se presentan hasta en un $30 \%$ de los casos ${ }^{6,11}$. Siontis y col. presentan una serie de 39 casos, 12 de éstos presentaron metástasis cerebrales durante su seguimiento, 4 de ellos presentes al momento del diagnóstico ${ }^{12}$, siendo el tiempo medio desde el momento del diagnóstico del sarcoma hasta la detección de metástasis cerebrales de 27 meses. Uno de los principales factores de riesgo es la ubicación del sarcoma, siendo más frecuentes en los ubicados al lado izquierdo.

Nuestro paciente debutó con tumor auricular, concordando con el grupo francés de sarcoma que describe la ubicación auricular más frecuente que la ventricu- 
lar, con predominio en aurícula derecha ${ }^{6}$. También se describe peor pronóstico en los sarcomas en esta ubicación, posiblemente por obstrucción y comportamiento "bulky"5,10,13. Con relación a las estadísticas chilenas, en la serie de González 4 de los 6 sarcomas tuvieron ubicación auricular izquierda ${ }^{2}$.

No existe laboratorio específico. Algunos reportes describen coagulación intravascular diseminada como presentación de angiosarcomas, que podría estar en contexto del fenómeno de Kasabach-Merritt (grave trombocitopenia, anemia hemolítica microangiopática y coagulopatía de consumo asociada a tumores vasculares). También se describe elevación de LDH, que podría indicar peor pronóstico, según Nakamura, y trombocitopenia que podría corresponder a mayor sheer stress ${ }^{14}$. El principal método diagnóstico es la ecocardiografía. Los elementos más sugerentes para diferenciar un mixoma de un sarcoma son: masas no adheridas a septum, múltiples masas, adherencia ancha a pared posterior izquierda, extensión a venas pulmonares, consistencia semisólida $^{13}$. El paciente descrito presentaba adherencia hacia posterior, extensión hacia venas pulmonares y múltiples masas. La RNM se ha masificado, adquiriendo relevancia en la caracterización de tumores cardía$\cos ^{11}$. De esta manera Kassi y colaboradores elaboraron

\section{Referencias}

1. BUTANY J, NAIR V, NASEEMUDDIN A, NAIR GM, CATTON C, YAU T. Cardiac tumours: diagnosis and manegement. Lancet Oncol. 2005; 6: 219E-228E. Disponible en https://www.thelancet.com/journals/lanonc/article/PIIS14702045(05)70093-0/fulltext (consultado 15 de agosto 2020).

2. GONZÁlEZ R, TOLOZA C, REYES R, SPENCER L, SEGUEL E, STOCKINS, et al. Tumores cardíacos primarios. Rev Med Chile 2020; 148: 327-335. un protocolo imagenológico comparado con histología, para determinar la malignidad con una precisión de un $100 \%$ para establecer benignidad versus un $92.3 \%$ para tumores malignos ${ }^{11}$.

El diagnóstico definitivo es histológico, pero no existe consenso en la clasificación de los tumores cardíacos malignos ${ }^{6}$. El angiosarcoma sería el más frecuente $(40 \%$ dentro de los sarcomas), luego el sarcoma pobremente diferenciado (23\%), fibrosarcoma y mixosarcoma (5\%), El rabdomiosarcoma y el leiomiosarcoma serían los menos prevalentes (menor 5\%) ${ }^{5}$. La histología marca un pronóstico en relación a la sobrevida de los pacientes con sarcoma cardíaco. El sarcoma de alto grado se asoció a peor sobrevida ${ }^{4,6}$. Otro factor de mal pronóstico es la presencia de metástasis 5,6 . Chen y colaboradores, mostraron que las metástasis más frecuentes fueron pulmonares $(46 \%)$, óseas $(17 \%)$ y cerebrales $(18 \%) 6$, éstas últimas asociadas a peores desenlaces $5,6,11$.

La terapia de elección es la resección quirúrgica y la sobrevida media fue 17.2 meses.

Lamentablemente, a pesar de una resección completa, estos tumores tienen una alta tasa de recidiva, con una sobrevida descrita entre 6-12 meses ${ }^{13}$. Otros manejos descritos son la radioterapia y quimioterapia.
3. OBRENOVIC-KIRCANSKI B, MIKIC A, PARAPID B, et al. A 30-year-single-center experience in atrial myxomas: from presentation to treatment and prognosis. Thorac Cardiovasc Surg. 2013; 61:530-536.

4. ALAM L, AGRAWAL K, KANKANALA V, FISHBERG R, POWELL D. Primary Cardiac Undifferentiated High-Grade Intimal Pleomorphic Sarcoma: A Case Series Report. Cardiol Res. 2020; 11:129-133. 
5. CHEN TW, LOONG HH, SRIKANTHAN A, ZER A, BARUA R, BUTANY. Primary cardiac sarcomas: A multi-national retrospective review. Cancer Med. 2019; 8: 104-110.

6. ISAMBERT N, RAY-COQUARD I, ITALIANO A, RÍOS A, KERBRAT P, GAUTHIER M, et al. Primary cardiac sarcomas: a retrospective study of the French Sarcoma Group. Eur J Cancer. 2014; 50:128-136.

7. RAMALINGAN R, MOORTHY N, RAO V, et al. Primary cardiac sarcoma presenting as shock. Indian J Thorac Cardiovasc Surg. 2009; 25: 31-33.

8. SIONTIS BL, ZHAO L, LEJA M, et al. Primary Cardiac Sarcoma: A Rare, Aggressive Malignancy with a High Propensity for Brain Metastases. Sarcoma. 2019;19: 593E-600E. Disponible en https://www.hindawi.com/journals/sarcoma/2019/1960593/ (consultado el 12 de agosto 2020).

9. REARDON MJ, DE FELICE CA, SHEINBAUM R, BALDWIN JC. Cardiac autotransplant for surgical treatment of a malignant neoplasm. Ann Thorac Surg. 1999; 67:1793-1795.
10. RANDHAWA JS, BUDD GT, RANDHAWA M, AHLUWALIA M, JIA X, DAW H, et al. Primary Cardiac Sarcoma: 25Year Cleveland Clinic Experience. Am J Clin Oncol. 2016; 39:593-599.

11. KASSI M, POLSANI V, SCHUTT RC, WONG S, NABI F, REARDON MJ, et al. Differentiating benign from malignant cardiac tumors with cardiac magnetic resonance imaging. J Thorac Cardiovasc Surg. 2019; 157: 1912-1922.

12. ABBAS A, GARFATH-COX KA, BROWN IW, SHAMBROOK JS, PEEBLES CR, HARDEN SP. Cardiac MR assessment of cardiac myxomas. Br J Radiol. 2015; 88:20140599.

13. NAKAMURA T, ASANUMA K, HAGI T, ¿SUDO A. Is Serum Lactate Dehydrogenase Useful for Predicting Oncological Outcome in Patients With Soft Tissue Sarcoma? Anticancer Res. 2019; 39: 6871-6875.

14. CHEN K. Fatal thrombocytopenia associated with intracardiac mass. J Cardiovasc Dis Res. 2012; 3: 65-66. 